\title{
Extensive and aggressive growth of adenoid cystic carcinoma in the lacrimal gland
}

\author{
Jonghyun Park, \\ Han Koo Kim, \\ Woo Seob Kim, \\ Tae Hui Bae \\ Department of Plastic and \\ Reconstructive Surgery, Chung-Ang \\ University Hospital, Chung-Ang \\ University College of Medicine, Seoul, \\ Korea
}

\begin{abstract}
Adenoid cystic carcinoma (ACC) in the lacrimal gland is a very rare disease with poor overall prognosis. Its primary treatment is surgical excision, including orbital exenteration and radical orbitectomy, which is combined with radiotherapy and chemotherapy. Age, histopathologic type, bone invasion, and tumor extent are known factors that affect the prognosis of ACC. Furthermore, perineural invasion is highly associated with local tumor recurrence and tumor base invasion. Here, we report a rare case of ACC in the lacrimal gland with superior sagittal sinus invasion that repeatedly recurred after the surgical excision.
\end{abstract}

Keywords: Adenoid cystic carcinoma / Lacrimal apparatus / Recurrence

\section{INTRODUCTION}

Malignant lacrimal gland tumor rarely occurs and results in poor overall prognosis. Its incidence rate is estimated to be 0.43 per million person-years [1]. Of all lacrimal gland tumors, $45 \%$ are malignant lesions, and adenoid cystic carcinoma (ACC) accounts for $60 \%$ of all malignant lesions; pleomorphic adenocarcinoma, $20 \%$ and primary adenocarcinoma, $10 \%$ [2]. The initial symptom of lacrimal gland tumors is mostly facial asymmetry due to displacement of the globe, commonly caused by lacrimal gland enlargement [3]. Pain is often suggestive of malignancy and may be secondary to bone or periorbital nerve involvement [4]. Computed tomography (CT) and magnetic resonance imaging (MRI) are the most important diagnostic imaging methods. The primary treatment of ACC is surgical removal, including orbital exenteration and radical orbitectomy, combined with radiotherapy and/or chemotherapy. However, the treatment of choice remains controversial because of the limited number of studies available given its rarity. Thus far,

\section{Correspondence: Han Koo Kim}

Department of Plastic and Reconstructive Surgery, Chung-Ang University

Hospital, 102 Heukseok-ro, Dongjak-gu, Seoul 06973, Korea

E-mail: hkkiim@cau.ac.kr

Received September 16, 2019 / Revised October 24, 2019 / Accepted November 22, 2019 only a few cases have been reported. Here, we describe a rare case of repeatedly recurring ACC in the lacrimal gland with extensive intracranial invasion, especially at the superior sagittal sinus.

\section{CASE REPORT}

A 29-year-old man presented with left upper eyelid swelling, which gradually increased in size over a few weeks before his first visit to the hospital. This non-tender swelling occurred 2 months before the first visit to the hospital for no conspicuous reason. The initial CT and MRI revealed a soft tissue mass $\left(2.7 \times 1.7 \mathrm{~cm}^{2}\right)$ with lobulated margin in the left lacrimal fossa (Fig. 1). Mass adhesion to the superior and lateral rectus muscle was suspected, and an irregular bony erosion on the adjacent lateral orbital rim was observed. Suspecting the possibilities of benign or malignant tumor, the patient underwent lacrimal mass excision and partial orbitectomy of the eroded lateral orbital wall while preserving the surrounding soft tissue for both diagnosis and treatment. The tumor already invaded the orbit intraoperatively; and upon biopsy, the patient was diagnosed with solid-type ACC in the lacrimal gland. Per patient's request, he was transferred to our hospital for further treatment. 


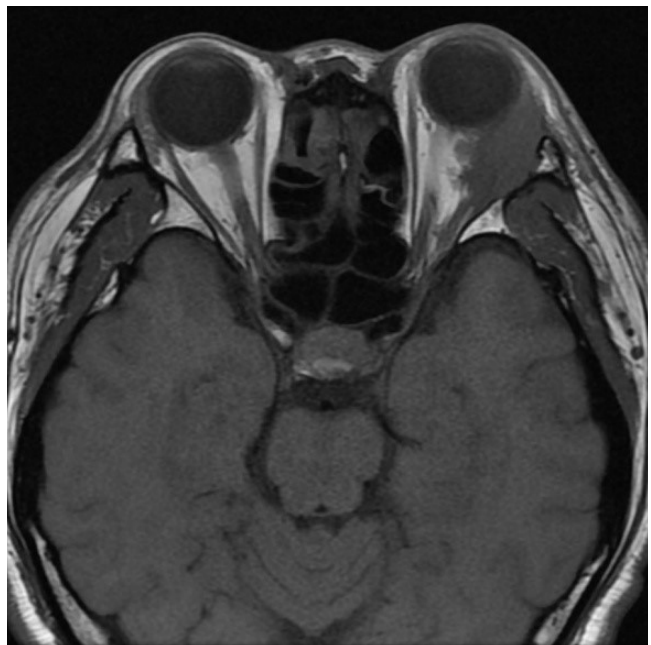

Fig. 1. Preoperative magnetic resonance imaging before the adenoid cystic carcinoma diagnosis.

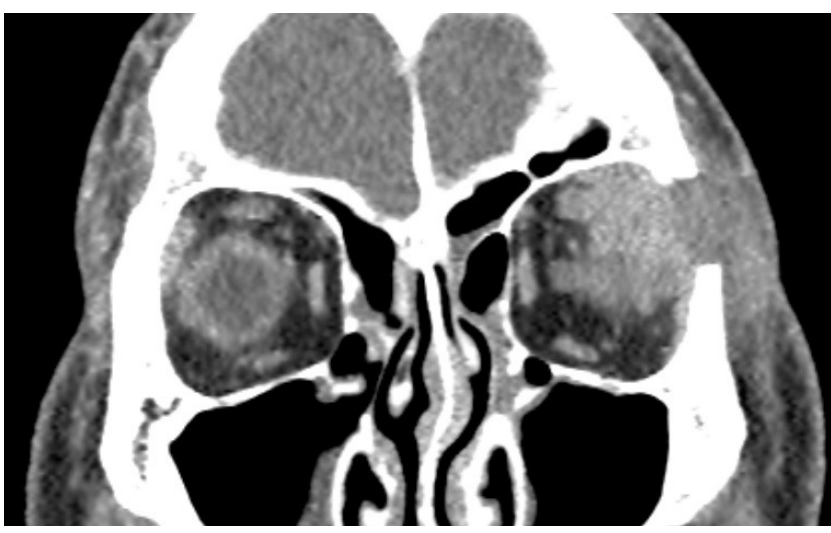

Fig. 2. Postoperative computed tomography after the first surgery.

The patient's follow-up CT images after the first operation showed a diffuse and enhanced soft tissue mass remaining in the superolateral orbit, extending to the superior orbital fissure without intracranial extension (Fig. 2). Decided by the ophthalmological department, the patient underwent radical tumor excision including exenteration of the globe and orbitectomy of the lateral orbital wall with safety margin cleared under frozen biopsies. The patient received 6,400 cGy of adjuvant radiotherapy postoperatively. No evidence of residual or recurrent tumor was observed on the patient's postoperative orbit CT and MRI scans at 4 and 8 months postoperatively.

Eleven months after the second surgery, on the regular followup appointment, the patient complained of swelling in his left forehead. MRI and CT scans showed an enhancement of the orbital apex, dural thickening in the right frontal to the left frontotemporal convexity and anterior falx, and suspicious tumor invasion in the anterior superior sagittal sinus without ab-

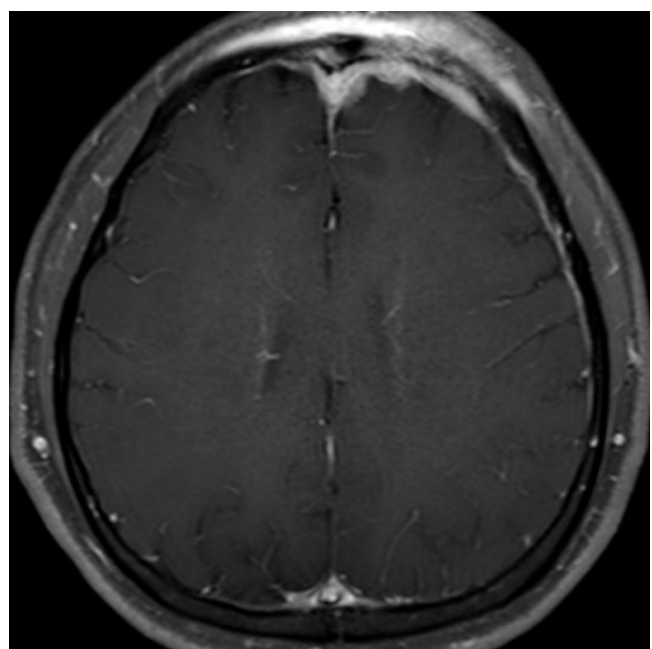

Fig. 3. Enhanced brain magnetic resonance imaging scan showing a well-enhanced nodular mass lesion in the anterior falx that was suspected to be a focal tumor invasion in the anterior superior sagittal sinus.

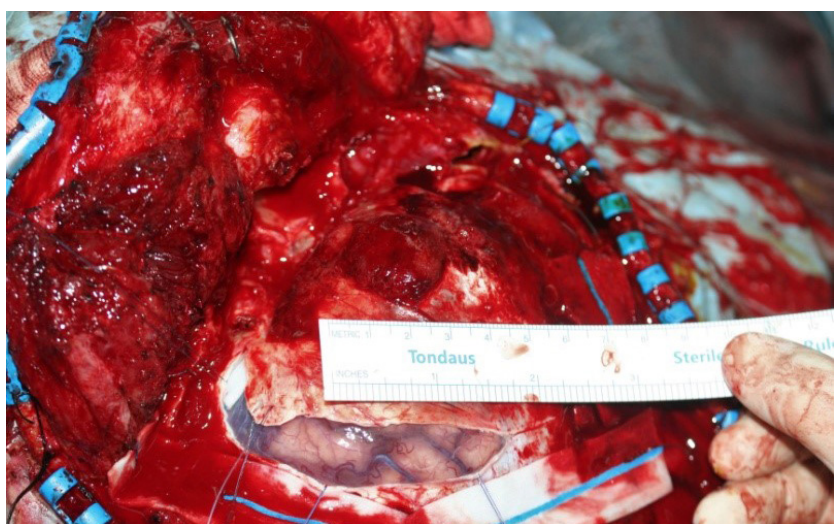

Fig. 4. Intraoperative photograph of the tumor mass firmly attached to the surrounding dura.

normal findings in the brain parenchyma and cerebrospinal fluid space (Fig. 3). Furthermore, lateral sphenoid bone involvement was observed. Considering the patient's medical history, recurrence of ACC was suspected and was confirmed by an incisional biopsy.

Per the multidisciplinary committee's decision, the patient underwent a third surgery, a second radical excision of the tumor including frontotemporoparietal craniotomy, and excision of the dura with tumor firmly attached on its surface (Fig. 4). The soft tissue around the orbital apex, surrounding the skin of the patient's left forehead and upper and lower lids, were excised, and an additional orbitectomy including total resection of the greater wing of the sphenoid bone was performed. After securing a clear margin with frozen biopsies, duraplasty and cranioplasty with a titanium mesh plate were performed. Anterolateral thigh chimeric flap containing a portion of the vastus lateralis muscle was used for reconstruction. The vastus lateralis muscle 

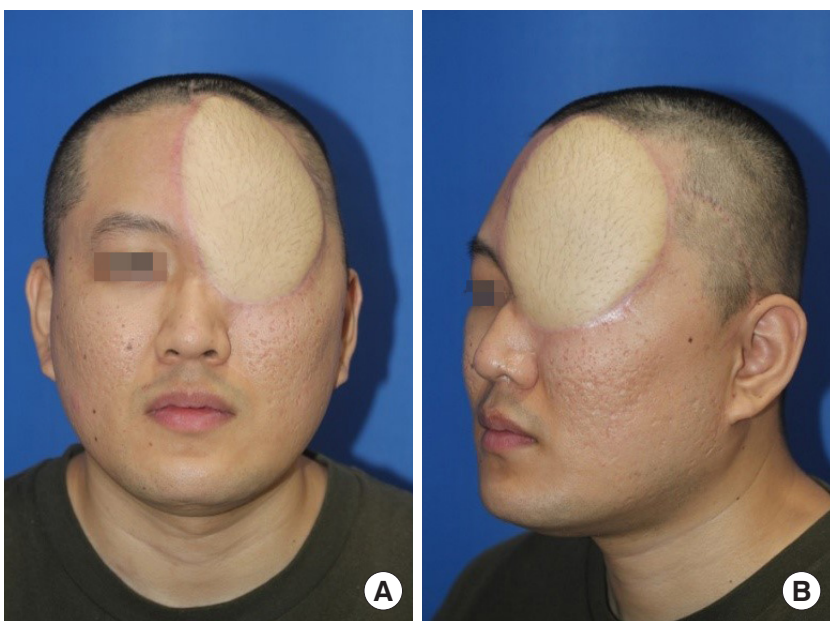

Fig. 5. Frontal (A) and oblique views (B).

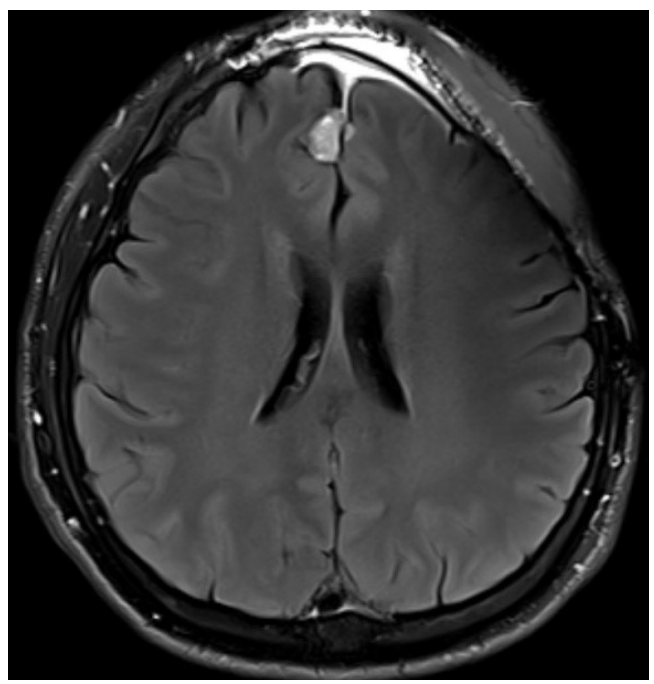

Fig. 6. Brain magnetic resonance imaging showing enhanced mass measuring $1.5 \mathrm{~cm}$ in diameter in the anterior falx.

was used to fill the space around the orbital apex. No specific postoperative complications were observed (Fig. 5). An additional 4,000 cGy of radiotherapy was administered 1 month postoperatively.

Regular follow-up imaging studies showed no residual tumor until 1 year after the third surgery, where MRI scan showed enhanced nodular mass lesion in the anterior falx and right temporal convexity (Figs. 6, 7). Multiple pulmonary nodules (measuring up to $4 \mathrm{~cm}$ ) on both hemithoraces were also observed in the patient's follow-up CT scans. Therefore, the patient was diagnosed with tumor recurrence and metastasis. Debulking surgery was performed including excision of the brain tumor and wedge resection of the lung.

Two and a half years after the first recurrence of the tumor, the patient is currently undergoing palliative care including

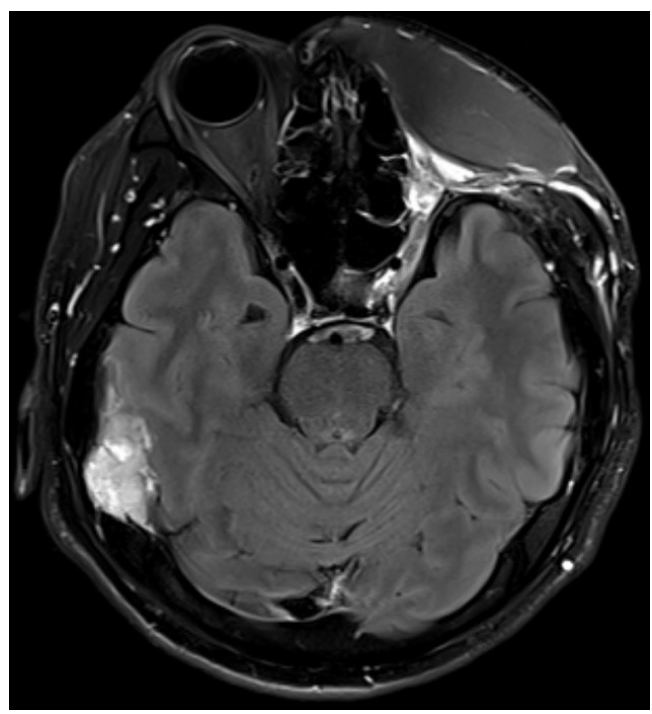

Fig. 7. Brain magnetic resonance imaging showing an irregular mass measuring $3.5 \mathrm{~cm}$ in diameter in the right temporal convexity with temporal bone involvement.

chemotherapy with vinorelbine, methotrexate, and bleomycin and has underwent an additional debulking surgery of the brain tumor as symptomatic treatment for headache and seizures.

\section{DISCUSSION}

Lacrimal gland ACC is usually aggressive and has a poor overall prognosis with a median overall survival of 7.6 years [5]. Age, histopathologic type, perineural invasion, bone invasion, and tumor extent are known factors affecting the prognosis of patients with ACC. Similar to the presented case, perineural invasion is highly associated with local tumor recurrence and tumor base invasion [6].

Although surgical intervention improves the overall survival, selecting the most effective surgical option remains controversial. Further, effects of intra-arterial cytoreductive chemotherapy (IACC) with cisplatin and doxorubicin before the surgery are currently investigated; however, this was not considered in this case because the patient had already undergone tumor excision on his first presentation to our hospital [7]. Orbital exenteration with/without removing the bony walls of the orbit is the standard surgical treatment. However, eye-sparing surgery and adjuvant radiotherapy have demonstrated favorable local control and long-term survival outcomes in patients with orbitconfined lacrimal gland ACC [8,9]. Ahmad et al. [10] have suggested that for carcinomas measuring $\leq 2.5 \mathrm{~cm}$ in the greatest dimension limited to the lacrimal gland or invading the periosteum of the lacrimal gland fossa, en bloc tumor excision can be 
cautiously considered an eye- and vision-preserving procedure. However, a recent study sparked a controversy by showing that eye-sparing tumor resection followed by adjuvant therapy was associated with high rates of local recurrence, metastasis, and death [11]. For carcinomas measuring $>2.5 \mathrm{~cm}$ or with neural invasion, orbital exenteration with orbitectomy is suggested to be the best surgical procedure, followed by radiotherapy [10].

The optimal management for lacrimal gland ACC remains unresolved and lacks general agreed-upon standard of care. The decision to remove the bone is usually prompted by either obvious radiographic evidence of bony involvement or intraoperative findings such as abnormal appearance of the bony walls in the orbit [10]. In this case, radical excisions, including orbital content exenteration, were not performed during the first operation before the ACC diagnosis. Exenteration and radical excision of the surrounding tissues were performed on the second surgery as a result of suspected invasion according to radiographic images. The tumor recurred even after removing all suspected lesions as well as the bony tissues, and the safety margin was cleared with a frozen biopsy.

In addition, although perineural invasion was not found on preoperative images, the recurring tumor at the superior sagittal sinus was firmly attached to the dural surface. ACC is known for its aggressive infiltration behaviors through the bone, perineural invasion with retrograde intracranial extension, and hematogenous or lymphatic spread, which result in a low survival rate of $<50 \%$ at 5 years and $20 \%$ at 10 years $[7,12]$. Kaur et al. [13] have suggested that lacrimal gland ACC may have an affinity for the dura and the expression of neural cell adhesion molecules of ACC may have caused perineural invasion possibly playing a role in the tumor recurrence in this case [14]. In light of these findings, although no agreed standard surgical treatment was available, ACC should be treated extensively. When faced with malignant lacrimal tumor or tumors suspicious of malignancy like the current case, an incisional biopsy should always be performed before the surgery. Diagnosing malignancy after the tumor excision may lower the chance of complete excision of malignant cells and even eliminate other treatment options like IACC. Furthermore, when performing orbitectomy, the normal bony tissue surrounding the malignant tumor should be removed along with the bony tissue involved in the tumor because of the aggressive characteristics of ACC.

In conclusion, our observations suggest that surgeons should aggressively treat lacrimal gland ACCs. Considering the aggressive nature of ACC, all surrounding tissues, including the normal bony tissue, should be removed. In cases like this, the range of clear safety margins and other treatments such as IACC can- not be determined prior to surgery because the patient presents after the excision of the primary tumor. To reduce tumor recurrence, careful planning of a more extensive, radical excision of the tumor is more recommended than when the patient presents with primary tumor in place.

\section{NOTES}

\section{Conflict of interest}

No potential conflict of interest relevant to this article was reported.

\section{Ethical approval}

The study was approved by the Institutional Review Board of Chung-Ang University Hospital (IRB No. 1907-014-16276) and performed in accordance with the principles of the Declaration of Helsinki. Written informed consent was obtained.

\section{Patient consent}

The patients provided written informed consent for the publication and the use of his images.

\section{ORCID}

Jonghyun Park https://orcid.org/0000-0001-9910-6945

Han Koo Kim https://orcid.org/0000-0002-2849-3973

Woo Seob Kim https://orcid.org/0000-0002-4104-3926

Tae Hui Bae https://orcid.org/0000-0002-0342-1439

\section{REFERENCES}

1. Hassan WM, Bakry MS, Hassan HM, Alfaar AS. Incidence of orbital, conjunctival and lacrimal gland malignant tumors in USA from Surveillance, Epidemiology and End Results, 19732009. Int J Ophthalmol 2016;9:1808-13.

2. Shields JA, Shields CL, Epstein JA, Scartozzi R, Eagle RC Jr. Review: primary epithelial malignancies of the lacrimal gland: the 2003 Ramon L. Font lecture. Ophthalmic Plast Reconstr Surg 2004;20:10-21.

3. Santos RR, Damasceno RW, de Pontes FS, Cursino SR, Nishiwaki-Dantas MC, Vital Filho J, et al. Ten-year follow-up of a case series of primary epithelial neoplasms of the lacrimal gland: clinical features, surgical treatment and histopathological findings. Arq Bras Oftalmol 2010;73:33-9.

4. von Holstein SL, Rasmussen PK, Heegaard S. Tumors of the lacrimal gland. Semin Diagn Pathol 2016;33:156-63.

5. Mallen-St Clair J, Arshi A, Tajudeen B, Abemayor E, St John M. Epidemiology and treatment of lacrimal gland tumors: a population-based cohort analysis. JAMA Otolaryngol Head Neck 
Surg 2014;140:1110-6.

6. Williams MD, Al-Zubidi N, Debnam JM, Shinder R, DeMonte F, Esmaeli B. Bone invasion by adenoid cystic carcinoma of the lacrimal gland: preoperative imaging assessment and surgical considerations. Ophthalmic Plast Reconstr Surg 2010;26:403-8.

7. Tse DT, Benedetto P, Dubovy S, Schiffman JC, Feuer WJ. Clinical analysis of the effect of intraarterial cytoreductive chemotherapy in the treatment of lacrimal gland adenoid cystic carcinoma. Am J Ophthalmol 2006;141:44-53.

8. Han J, Kim YD, Woo KI, Sobti D. Long-term outcomes of eyesparing surgery for adenoid cystic carcinoma of lacrimal gland. Ophthalmic Plast Reconstr Surg 2018;34:74-8.

9. Wolkow N, Jakobiec FA, Lee H, Sutula FC. Long-term outcomes of globe-preserving surgery with proton beam radiation for adenoid cystic carcinoma of the lacrimal gland. Am J Ophthalmol 2018;195:43-62.

10. Ahmad SM, Esmaeli B, Williams M, Nguyen J, Fay A, Woog J, et al. American Joint Committee on Cancer classification predicts outcome of patients with lacrimal gland adenoid cystic carcinoma. Ophthalmology 2009;116:1210-5.

11. Yang J, Zhou C, Wang Y, Fan X, Jia R. Multimodal therapy in the management of lacrimal gland adenoid cystic carcinoma. BMC Ophthalmol 2019;19:125.

12. Falcone MM, Tran KD, Tavakoli M, Ortiz DF, Barredo JC, Lee WW. Adenoid cystic carcinoma of the lacrimal gland in a 14-year-old male. Orbit 2017;36:448-51.

13. Kaur A, Harrigan MR, MeKeever PE, Ross DA. Adenoid cystic carcinoma metastatic to the dura: report of two cases. J Neurooncol 1999;44:267-73.

14. Shang J, Sheng L, Wang K, Shui Y, Wei Q. Expression of neural cell adhesion molecule in salivary adenoid cystic carcinoma and its correlation with perineural invasion. Oncol Rep 2007; 18:1413-6. 